\title{
A Rare Case of Fish Bone Penetrating Injury to Radius Causing Osteitis and Non-Healing Sinus*
}

\author{
Paragjyoti Gogoi $^{1 \#}$, Krishanu Kakati ${ }^{1}$, Sunil Poonia ${ }^{1}$, Aditi Das ${ }^{2}$ \\ ${ }^{1}$ Department of Orthopaedics \& Trauma, Silchar Medical College, Silchar, India; ${ }^{2}$ Department of Radio-Diagnosis, Silchar Medical \\ College, Silchar, India. \\ Email: \#pggogoiparag@gmail.com
}

Received July 25 ${ }^{\text {th }}, 2013$; revised August 21 ${ }^{\text {st }}, 2013$; accepted August $28^{\text {th }}, 2013$

Copyright @ 2013 Paragjyoti Gogoi et al. This is an open access article distributed under the Creative Commons Attribution License, which permits unrestricted use, distribution, and reproduction in any medium, provided the original work is properly cited.

\begin{abstract}
Penetrating injury to radius or any other bone by fish bone is a rare kind of injury. Commonly metallic sharp objects, bullets, sharpnels, glass particles, hard wooden pieces are the causes of penetrating injury to bones. Radio-opaque objects are easy to detect and thus retrieval is relatively simpler than the radiolucent objects. Non-biological foreign bodies do not incite any reaction in the host bone whereas biological foreign bodies of either plant or animal origin cause hypersensitivity reaction. Osteitis along with excessive formation of new bones may be presented as a part of host reaction to the foreign body. We are presenting here an interesting case of a fisherman boy who sustained penetrating injury to radius by fish bone resulting in osteitis and a chronic non-healing sinus with watery discharge.
\end{abstract}

Keywords: Fish Bone; Foreign Body; Granuloma; Penetrating Injury; Radio-Opaque

\section{Introduction}

Foreign body retained in the soft tissues of extremities causing sinus formation is not uncommon. Mostly the working class people are affected. Wooden foreign body, blackthorn injury, glass particle, metallic pieces are the major causes of granuloma formation along with sinus [1-3]. A case of fisherman penetrating injury by fish bone along with granuloma formation is reported [4]. However, fish bone penetrating the bone causing osteitis and granuloma formation is very rare. We are reporting a very unusual case of fish bone injury to forearm where the fish bone penetrated the radius and there were osteitis and granuloma formations along with a sinus. Perhaps this is the first case of this kind. The purpose of this paper is to highlight the uniqueness and the rarity of this case and to bring to the fore the necessary discussion in management of medical and surgical oddities.

\section{Case Report}

A 14-year-old boy attended our outpatient department with pain over left forearm and a discharging sinus on the volar aspect. He gave a peculiar history of sustaining

\footnotetext{
*Conflict of Interests: The authors declare that they have no conflict of interests. Consent: Informed consent was taken for this work.

${ }^{\#}$ Corresponding author.
}

trauma over his left forearm. He hailed from a fisherman family and while helping his father out in transferring big fishes from one container to another he fell down over a big fish and sustained injury over his left forearm. There was a pricking injury which healed after primary treatment and some medications. He was normal for four months except some catch in some rotational movement. Gradually he started having pain over the forearm along with slight swelling. Within two weeks of pain, he developed a sinus over the volar aspect of left forearm near the old pricking site with some watery discharge.

On examination a scarred area was noted over the volar aspect of mid-forearm with a small sinus with watery discharge (Figure 1). The sinus seemed to be adhered to underlying soft tissues and mild tenderness on palpation was noted. Range of movement of elbow and wrist were within normal limits. There was mild pain at the extremes of pronation and supination of the forearm.

$\mathrm{X}$-rays of the forearm revealed a radio-opaque linear foreign body impinging the radius and surrounded by new bone formation and a lytic radio-lucent ream (Figures 2 and 3). Marked osteitis with extensive periosteal reaction is noted both proximal and distal to the foreign body. Musculo-skeletal ultrasonography using high frequency probe confirmed the foreign body along with formation of a granulomatous mass surrounding it (Fig- 


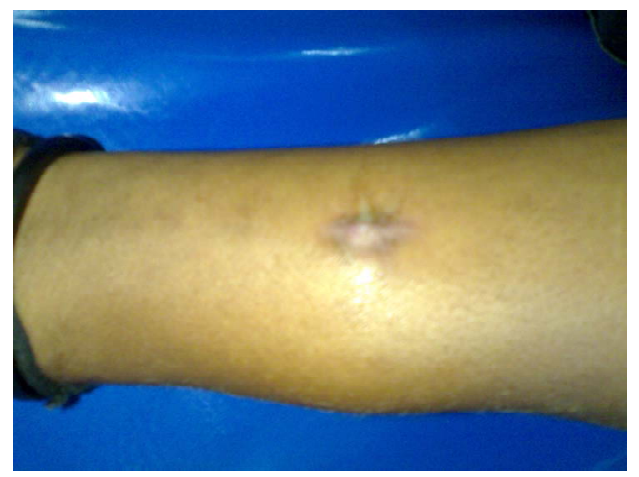

Figure 1. Sinus formation in forearm.

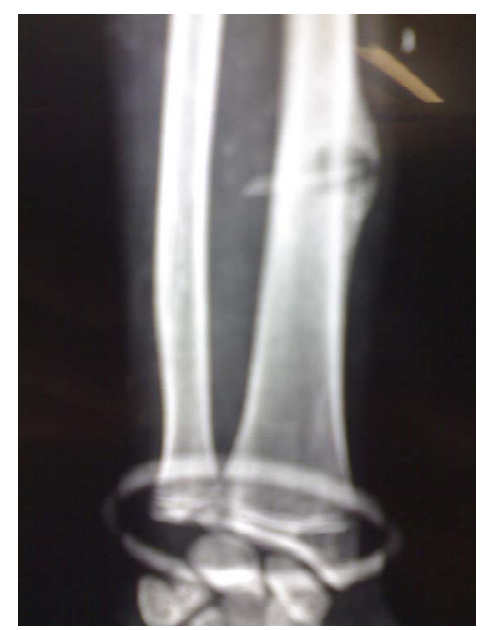

Figure 2. AP view showing fish bone and osteitis of radius with new bone formation.

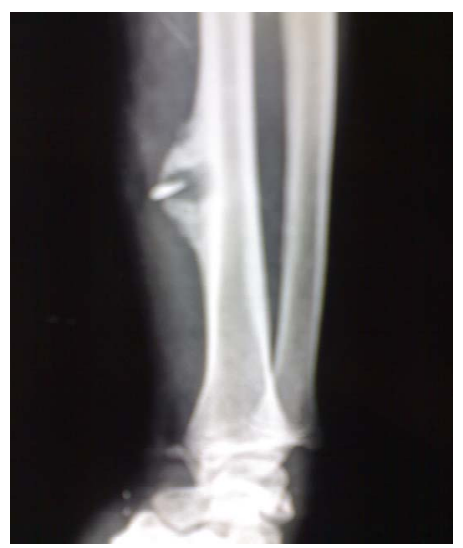

Figure 3. Lateral view showing radio-luceny around the fish bone and periosteal reaction of radius.

ures 4 and 5). There was no injury to the neuro-vascular structures.

Blood parameters revealed leucocytosis with eosinophilia and ESR was mildly elevated. Chest X-ray was normal.

From the clinical history and radiological pictures the diagnosis of retained foreign body impinging the radius with osteitis was made and decision taken to explore the lesion.

The lesion was addressed by anterior Henry's approach. The sinus was excised. The area was exposed well with due care and the fish bone identified impinging the radius. There was necrotic area around the fish bone covered by a fibrotic pseudo-capsule. The fish bone removed in single piece and measured $28 \mathrm{~mm}$ with a sharp pointing end (Figures 6 and 7). The fibous pseudocapsule surrounding the fish bone was excised (Figure 8). Extensive new bone formation was noted in the radius around the impinged area. There was a central crater filled with necrotic material surrounded by new bones (Figure 9). The new bones were chiseled out to get a smooth contour of the radius (Figure 10). Thorough curettage of the area was done and the material sent for histology. Histological picture of the curetted material was consistent with those of a granuloma.

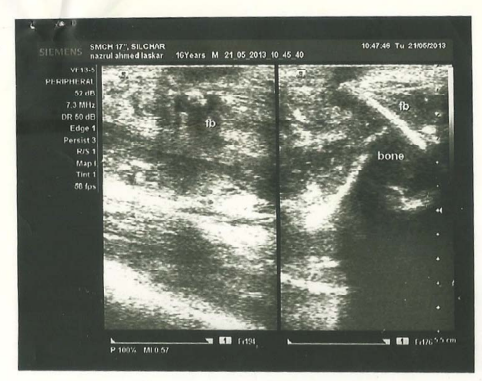

Figure 4. Ultrasonography of forearm.

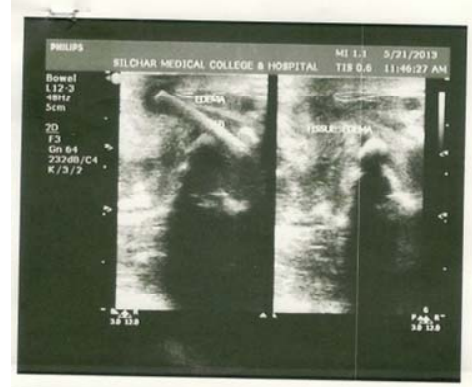

Figure 5. USG showing embedded foreign body.

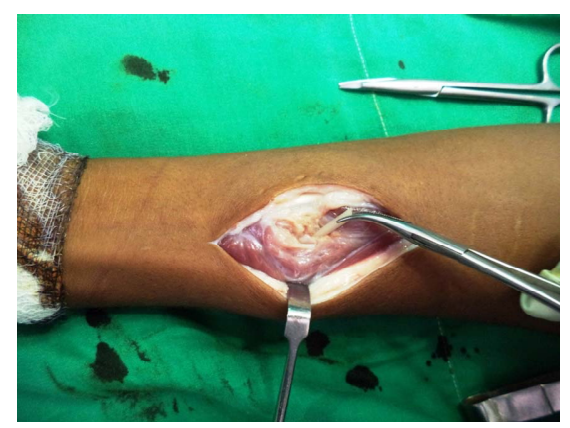

Figure 6. Fish bone removed in toto. 


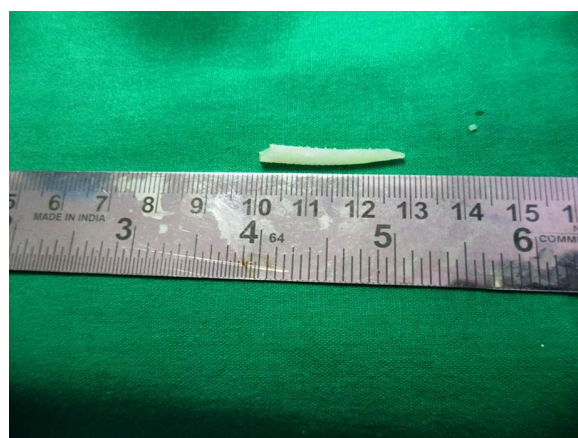

Figure 7. Fish bone measuring $28 \mathrm{~mm}$.

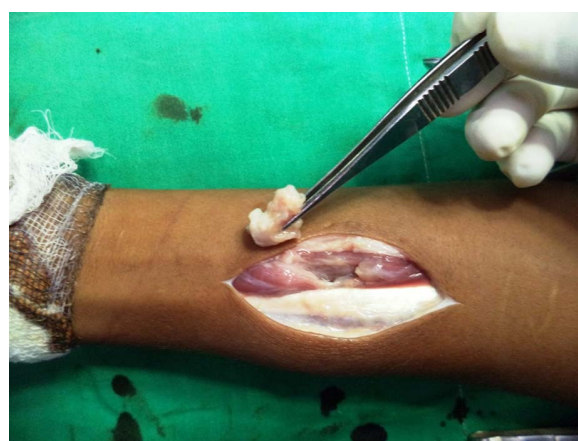

Figure 8. Excised fibrous pseudocapsule.

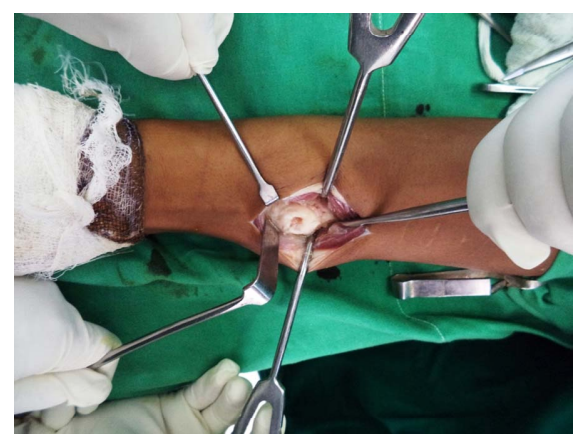

Figure 9. Crater on the radius surrounded by new bones.

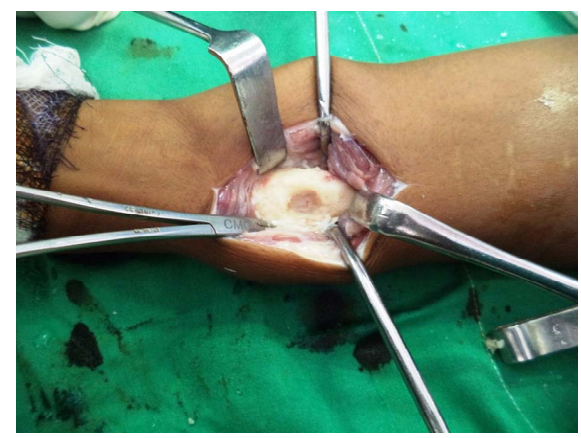

Figure 10. Excessive new bone chiseled out.

Post-operative X-rays confirmed thorough removal of the foreign body and removal of new bones (Figure 11).

The surgical wound healed well and the boy got rid of the symptoms. He was followed up for six months and no

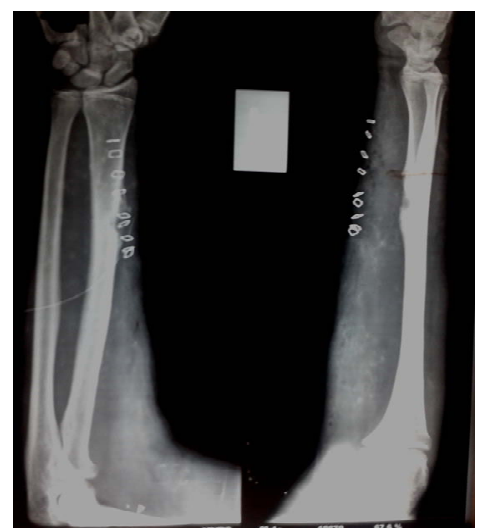

Figure 11. Post operative X-ray.

recurrence of sinus was there.

\section{Discussion}

Fishing is regarded as one of the oldest professions. Many people derive their livelihood from fishing. It is also seen as a great recreational activity. But this is also not free from injury risks like many other professions. Mostly injury occurs due to penetrating injury by the fins to the extremities $[4,5]$. In some rare instances penetrating injury to heart can occur by long stings of some species [6]. Some fishes contain venom in their stings and fish envenomatosis require special care and treatment. Apart from broad spectrum antibiotics, tetanus toxoid, analgesics, this condition may demand specific antivenom administration [7].

There have been many case reports on fish bone injuries to hands and forearm as is evident in the study carried out by Imberg R. et al. [4] in which 116 patients were followed up with injuries sustained by fish bone to their hands and forearm. Strangely enough not a single case reported embedding of the fish bone to bone. To the best of our knowledge the above mentioned study is the most extensive one carried out in this matter.

Diagnosis of retained fish bone is not difficult. There is always a history of penetrating injury resulting a skin wound. Irrespective of the history it should be presumed that there is a part of fish bone inside the body until all investigations are over. X-ray usually detects the radioopaque fish bones. In case of radio-lucent objects ultrasonography is helpful $[1,8]$. Normally CT scan or MRI scan is not required.

Inoculation of polymicrobial flora following fish bone injury is a concern. There are reports of wound contamination by unusual species like vibrio, actino-mycosis etc. [9,10]. Broad spectrum antibiotics should always be recommended for such injury. Tetanus toxoid also may have a role in preventing life threatening toxaemia.

Retained foreign bodies in the soft tissues incite granulomatous reaction. The host tries to isolate the biological 
foreign body by forming a granuloma around it [8]. In the centre of the granuloma there lies the foreign body and a layer of necrotic tissues surrounding it.

Penetration of the host bone by fish bone is a rare event. There is a host reaction in the form of osteitis or periosteal reaction. There is formation of excessive bone surrounding the fish bone which is akin to granuloma formation in soft tissues. In this particular case the fish bone was long enough to have partly inside the radius and partly in soft tissues of forearm. Therefore we found excessive fibrous tissues surrounding the fish bone in the soft tissue part and excessive bone formation around it in the bony tract. The rareness of this case and the relative dearth of literature in this matter compelled us to direct our treatment towards normal protocol of granulamatous infections of bone. The persistence of infection for four months and the osteitis led us to take the decision of surgical exploration immediately and excision of the sinus tract along with curettage of the bone.

\section{Conclusion}

In penetrating injury by fish, the possibility of the retained part inside the body should always be suspected. The presence of sinus reconfirms this suspicion. X-ray is the most commonly used noninvasive investigations to detect radio-opaque foreign bodies. Ultrasonography detects the presence of radiolucent objects and highlights the soft tissue conditions like granuloma formation, presence of abscess and exact location. Surgical removal as well as excision of the granulomatous sinus tract is the rule. Broad spectrum antibiotics help in rapid healing and can also cover the unusual pathogens.

\section{REFERENCES}

[1] B. Borgohain, N. Borgohain, A. Handique and P. J. Gogoi, "Case Report and Brief Review of Literature on Sonographic Detection of Accidentally Implanted Wooden Foreign Body Causing Persistent Sinus,” Critical
Ultrasound Journal, Vol. 4, No. 1, 2012, p. 10. doi:10.1186/2036-7902-4-10

[2] H. Sharma and A. D. Meredith, "Blackthorn Injury: A Report of Three Interesting Cases,” Emergency Medicine Journal, Vol. 21, No. 3, 2004, p. 392. doi:10.1136/emj.2002.002782

[3] C. K. Lee, T. Sara Ahmad and B. J. J. Abdullah, "Splinter Removal with the Aid of Ultrasonography: A Case Report,” Malaysian Orthopaedic Journal, Vol. 2, No. 2, 2008, pp. 47-49.

[4] R. Imberg, I. Potasman, Y. Weissman and M. Grupper, "Hand Infections Following Penetrating Fish Fins or Bones Injuries (FFBI): A Large, Hospital Based, Retrospective Study,” Infection, Vol. 36, No. 6, 2008, pp. 565569.

[5] S. Saraf, "An Unusual Foreign Body in the Forearm: A Case Report,” The Internet Journal of Hand Surgery, Vol. 5, No. 1, 2013.

[6] V. Haddad, R. A. de Souza and P. S. Auerbach, "Marine Catfish Sting Causing Fatal Heart Perforation in a Fisherman,” Wilderness, Vol. 19, No. 2, 2008, pp. 114118.

[7] J. Y. Lee, L. C. Teoh and S. P. Leo, "Stonefish Envenomations of the Hand-A Local Marine Hazard: A Series of 8 Cases and Review of the Literature," Ann Acad Med Singapore, Vol. 33, No. 4, 2004, pp. 515-520.

[8] A. Akira, H. Masahito, H .Yoshihiro, I. Shuji and I. Eiji, "Imaging Features of Foreign Body Granuloma in the Lower Extremities Mimicking a Soft Tissue Neoplasm," Upsala Journal of Medical Sciences, Vol. 114, No. 1, 2009, pp. 46-51. doi:10.1080/03009730802602455

[9] C. H. Chang-Chien, H. T. Ding, C. Liu and C. S. Yang, "Vibrio Infection Associated with Finning Injury of the Hand,” Injury, Vol. 38, No. 5, 2007, pp. 614-618.

[10] D. A. Hudson and T. M. de Chalain, "Hand Infections Secondary to Fish Bone Injuries," Annals of The Royal College of Surgeons of England, Vol. 76, No. 2, 1994, pp. 99-101. 